\title{
Modelling the Track Allocation Problem in Railway Stations
}

\author{
Wei Liu ${ }^{1, a}$, Xiaoning $\mathrm{Zhu}^{2, \mathrm{~b}}$ and Liujiang Kang ${ }^{3, \mathrm{c} *}$ \\ ${ }^{1}$ School of Traffic and Transportation, Beijing Jiaotong University, Beijing, China. \\ ${ }^{2}$ School of Traffic and Transportation, Beijing Jiaotong University, Beijing, China. \\ ${ }^{3}$ School of Traffic and Transportation, Beijing Jiaotong University, Beijing, China. \\ a13114210@bjtu.edu.cn, bxnzhu@bjtu.edu.cn, ${ }^{\mathrm{b}}$ ljkang@bjtu.edu.cn
}

Keywords: Railway, Track allocation problem, Bottleneck section, Stochastic schedule, Simulated annealing algorithm.

Abstract. Track allocation problem in railway stations with stochastic viewpoint is a crucial part of the planning processes for railway companies. This paper proposes a stochastic schedule based optimization model to solve the station track allocation problem that considers minimum occupation time of station reception-departure lines. The stochastic schedule consists of the average and variance of train arrival times and departure times, respectively. Owning to the NP-Completeness of the problem, a simulated annealing algorithm is designed to solve the mathematical programming model.

\section{Introduction}

The railway system is a large and complex system which contains many working processes that relate to technical facilities, railway transport organization system, train operation organization, human labors and the external environment.

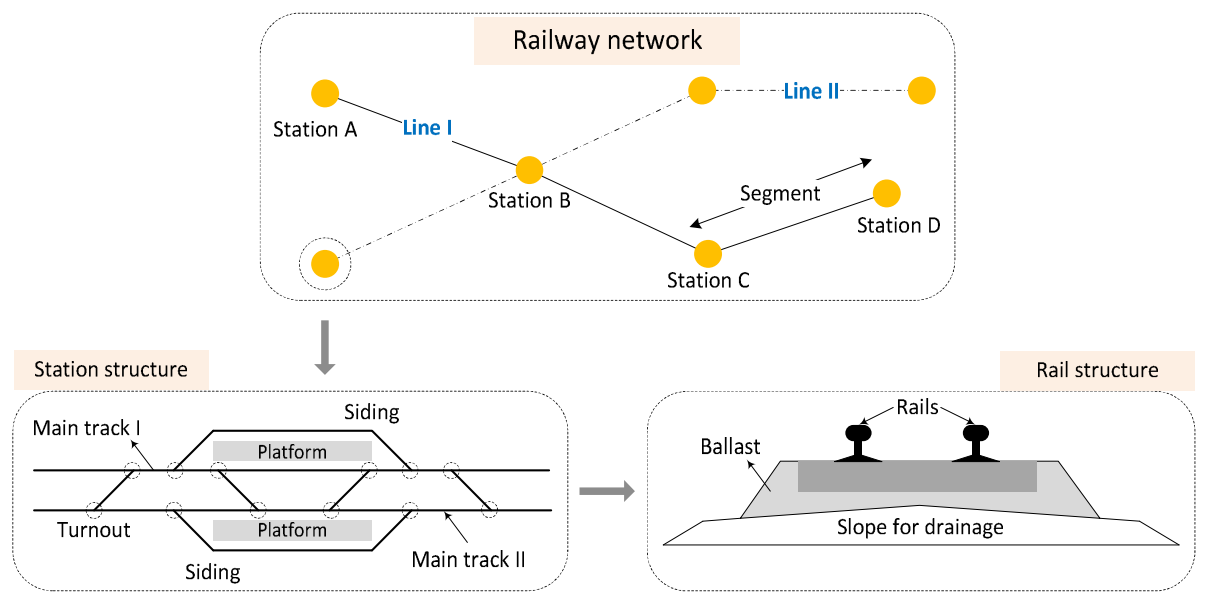

Fig. 1 Illustration of a simple railway network

Fig. 1 shows a railway network which consists of seven railway stations and six segments. Railway stations are the places where trains can dwell to make passengers get on, get off and transfer. Apart from main tracks, stations also have siding tracks to facilitate trains passing and overtaking. In addition, tracks which consist of rails, ballast, ties, etc., are the roadways of a railway system. Clearly, railway stations are the key nodes in railway networks; therefore, efficiently running of trains in a station is crucial in the working processes to railway companies. While in practical operations, trains often miss the original schedules affected by unexpected accidents and many other uncertain factors, e.g., signal failures and turnouts breaking down. To consider the uncertainty, track allocation problem in railway stations is studied with stochastic viewpoint that adopts unfixed train schedules to solve the uncertain arrival/departure times of trains at/from stations. 


\section{Mathematical Models and Methodology}

The track allocation problem is about assigning a platform to each coming train, considering suitable in-route and out-route. Route choices lead to different route durations and imply different in-route-begin and out-route-end times. As we can see from Fig. 2, a train goes the red path that consists of three in-route turnouts, track I, and two out-route turnouts. The total duration time of turnouts is $\sum_{i=1}^{5} t_{i j}$, where $t_{i j}$ represents the time of train $j$ that occupies turnout $i$. Considering the unfixed train arrival and departure times, we use $\tau_{r j} \%$ and $\tau_{r j} \%$ to represent the real starting and ending time of train $j$ that occupies track $r$. Therefore, equation $(\% \%-\%$ i $\%$ can be formulated as the duration time of train $j$ on track $r$. This paper aims to address the track allocation problem based on the stochastic schedule.

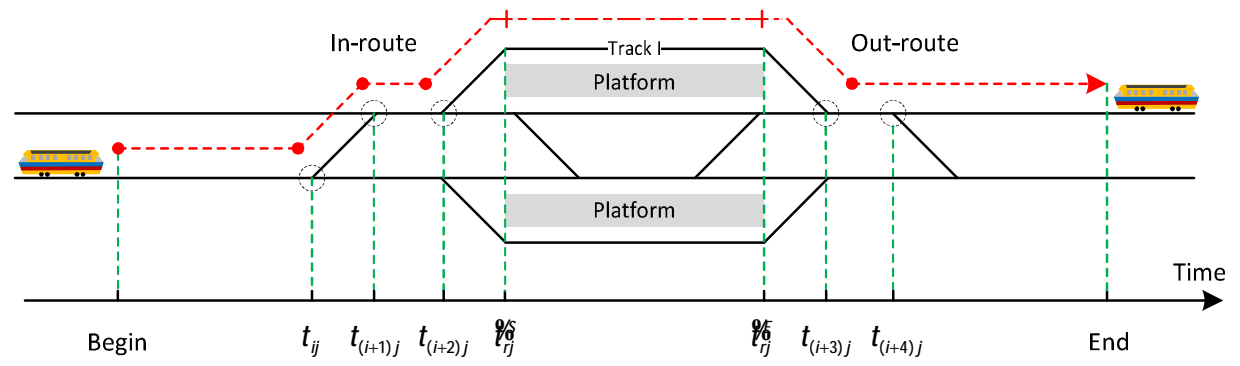

Fig. 2 Train path in a passenger station

In railway stations, a coming or leaving train will stay on or pass a reception-departure track and traverse several GTs. Because of the limited track resources and bottleneck capacity, they have to be allocated to trains under some solid rules and constraints.

In general, train arrival and departure times are determined by the TWD. However, because of kinds of operation accidents and emergency incidents, trains do not always obey the schedule strictly, which makes the track occupation time of each train random and unknown. The following Eq. 1 and Eq. 2 describe the minimum occupation time in station tracks, where $t_{r j}$ represents the time of train $j$ that uses track $r$, and $x_{r j}$ denotes a 0-1 variable. If train $j$ occupies track $r, x_{r j}=1$. Otherwise, $x_{r j}=0$.

$$
\begin{gathered}
\min Z_{1}=\sum_{j=1}^{m} t_{r j} \cdot x_{r j} . \\
t_{r j}=q_{r j} \% t_{r j} .
\end{gathered}
$$

The real train departure time, ${ }_{r j} \%$, is calculated by its average departure time and variance of departure time, where $E\left(i_{r j} / \%\right)$ and $\delta\left(i_{r j}\right)$ are counted by statistical experiments. The real train arrival time, $t_{r j}$, is obtained by the same method. Thus, the real ending and starting occupation times of station reception-departure lines could be tracked as follows, where $\lambda_{1}$ and $\lambda_{2}$ are weight parameters.

$$
\begin{aligned}
& q_{r j} F_{0}=E(q \%)+\lambda_{r j} \cdot \delta\left(q_{r j} \%\right) . \\
& f_{r j}=E(f \%)+\lambda_{2} \cdot \delta(f \%) \text {. }
\end{aligned}
$$

Besides, several operating constraints in passenger stations are given to set limits on track occupations. To fulfill the service as well as enable operating safety, the following constraints are proposed to avoid collision [1].

(I). Uniqueness occupation of each track at one time. Each reception-departure track could be utilized by only one train at most before available to the next train [1]. Therefore, Eq. 5 is given as: 


$$
\sum_{j=1}^{m} x_{r j} \leq 1
$$

(II). Each arrival train should occupy one and only one track. Once the train is allocated to a fixed track, it cannot transfer to the others [1]. Therefore, Eq. 6 should be satisfied..

$$
\sum_{r=1}^{l} x_{r j}=1
$$

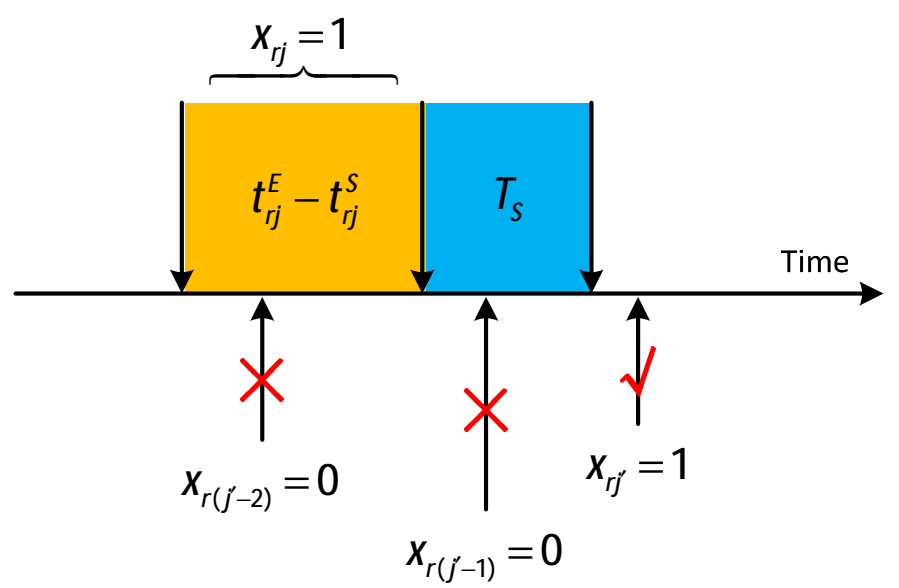

Fig. 3 The security interval time

The safety interval time $T_{S}$ is the minimum interval period for two continuous trains stopping on one same track that should be satisfied, see Eq. 7. Thus, train $\left(j^{\prime}-1\right)$ also cannot stop on track $r$. Consequently, only train $j^{\prime}$ can stay on track $r$ in Fig. 3. Here, binary variable $x_{r j^{\prime}}$ is introduced by Eq. 7 for all $r \in R, j \in J$ and $j^{\prime} \in J$,

$$
M \cdot\left(x_{r j^{\prime}}-1\right) \leq q_{r j} q_{r j} F_{r j}-T_{S} \leq M \cdot x_{r j^{\prime}}
$$

where $M$ is a very large positive value. Eq. 7 ensures that $x_{r r^{\prime}}$ equals 1 only if train $j^{\prime}$ arrives with enough late gap so that train $j^{\prime}$ can stop on track $r$.

Our model aims to seek the minimum occupation time of station tracks. Therefore, the model can be described as follows:

$$
\begin{gathered}
\min Z_{1}=\sum_{j=1}^{m}\left(\frac{\rho \%}{f_{r j}}-\frac{\rho \%}{r j}\right) \cdot x_{r j} . \\
\text { s.t. }\left\{\begin{array}{l}
\sum_{j=1}^{m} x_{r j} \leq 1, \quad r=1,2, \mathrm{~L}, l \\
\sum_{r=1}^{l} x_{r j}=1, \quad j=1,2, \mathrm{~L}, m \\
M \cdot\left(x_{r j}-1\right) \leq \% \%_{r j}-q_{r(j-1)}-T_{S} \leq M \cdot x_{r j}
\end{array}\right.
\end{gathered}
$$

The SA algorithm below aims at searching for the optimum solutions of the track allocation problem in railway stations. The detailed algorithmic steps are given as following:

Step 1: Initialization

Set SA parameters: starting temperature $T_{0}$, stopping temperature $\tau$, cooling coefficient $\omega$ and Markov Length $L$. Input TWD (timetable), bottleneck map of the station, GT time and safety interval time $T_{S}[3-7]$.

Let temperature $T=T_{0}$. Produce a solution $s_{0}$ randomly and calculate the corresponding objective value $f^{(0)}$, displaying it.

Check constraints (5-7). If yes, turn to step 1.4; otherwise, go back to step 1.2. 
Set the outer iteration $\eta=0$.

Step 2: For the current temperature T, check 2.1-2.4

Set the inner iteration counter $\kappa=1$.

Let $\eta=\eta+1$. Generate a new solution $s_{1}$ by exchanging two trains' tracks randomly.

Check constraints (5-7). If yes, turn to step 2.4; otherwise, go back to step 2.2.

Calculate the objective value $f^{(\eta)}$.

Calculate $\Delta f$, where $\Delta f=f^{(\eta)}-f^{(\eta-1)}$. If $\Delta f \leq 0, s_{1}$ replaces $s_{0}$, and display $f^{(\eta)}$; otherwise let $s_{1}$ replace $s_{0}$ with the probability $\rho, \rho=\exp (-\Delta f / T)$, and display $f^{(\eta)}$.

If $\kappa=L$, go to Step 3; otherwise set $\kappa=\kappa+1$, and return to 2.2.

Step 3: Stop or not

If $T \leq 0.01$, stop. Otherwise $T=T \times 0.95$, and return to Step 2 .
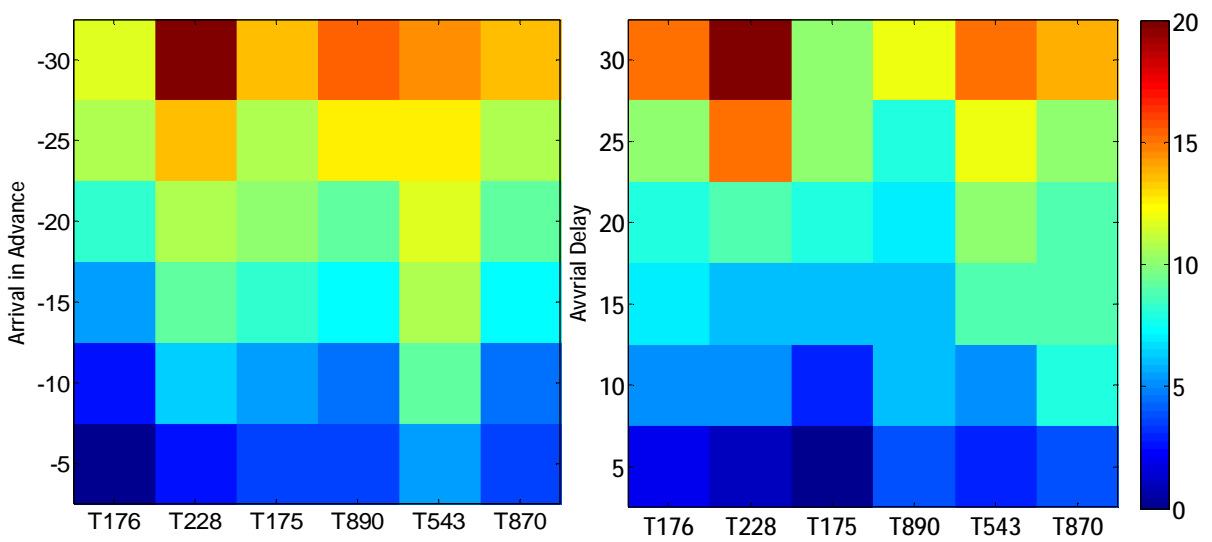

Fig. 4 Effects of train arrival unpunctually

\section{Analysis of Train Arrival in Advance and Arrival Delay}

In this section, we detect the effects of unpunctual train-arrival times on total occupation time of station reception-departure lines. Floating train arrival times are tested from 5 to $30 \mathrm{~min}$ for the below six trains, respectively, see Fig. 4. In the scenario of train arrival in advance, we find that train T228 influences the total occupation time of station tracks most. Meanwhile, it indicates that train T228 also influences the track occupation most for the delay scenario.

\section{Conclusions}

This paper proposes a stochastic schedule based optimization model to solve the station track allocation problem that considers minimum occupation time of station reception-departure lines. The stochastic schedule consists of the average and variance of train arrival times and departure times, respectively. Owning to the NP-Completeness of the problem, a simulated annealing algorithm is designed to solve the mathematical programming model.

\section{Acknowledgements}

This paper is supported by the NSFC (No.71473259, 71390332), and the Specialized Research Fund for the Doctoral Program of Higher Education (No. 20130009110001).

\section{References}

[1] J.J. Wu, L.J. Kang, H.J. Sun and X.L. Jia, Track allocation optimization in railway station: mean-variance model and case study, Journal of Transportation Engineering. 139 (2013) $540-547$. 
[2] L.J. Kang, X.N. Zhu, J.J. Wu, H.J. Sun, S. Siriya, and T. Kanokvate, Departure time optimization of last trains in subway networks mean-variance model and GSA algorithm, Journal of Computing in Civil Engineering. 041401 (2014) 1-12.

[3] L.J. Kang, J.J. Wu and H.J. Sun, Using simulated annealing in a bottleneck optimization model at railway stations, Journal of Transportation Engineering. 138 (2012) 1396-1402.

[4] L.J. Kang and X.N. Zhu, A Simulated Annealing Algorithm for First Train Transfer Problem in Urban Railway Networks, Applied Mathematical Modeling. doi:10.1016/j.apm.2015.05.008.

[5] L.J. Kang, J.J. Wu, H.J. Sun, X.N. Zhu and Z.Y. Gao, A case study on the coordination of last trains for the Beijing subway network, Transportation Research Part B. 72 (2015) 112-127.

[6] L.J. Kang, J.J. Wu, H.J. Sun, X.N. Zhu and B. Wang, A practical model for last train rescheduling with train delay in urban railway transit networks, Omega. 50 (2015) 29-42.

[7] W. Liu, X.N. Zhu, and L.J. Kang, Real-time Track Reallocation for Emergency Incidents at Large Railway Stations, Mathematical Problems in Engineering. 296394 (2015) 1-11. 